\title{
USO DEL PLASMA RICO EN FACTORES DE CRECIMIENTO AUTÓLOGO EN CIRUGÍA ORAL Y MAXILOFACIAL: PATOLOGÍA TUMORAL
}

\author{
USE OF RICH PLASMA IN AUTOLOGOUS GROWTH FACTORS IN ORAL \\ AND MAXILLOFACIAL SURGERY: TUMOR PATHOLOGY
}

USO DE PLASMA RICO EM FATORES AUTOLÓGICOS DE CRESCIMENTO EM CIRURGIA ORAL E MAXILLOFACIAL: PATOLOGIA TUMORAL

\section{JORGE BARONA TERÁN, PAMELA MOREIRA ZEVALLOS}

Universidad Católica de Santiago de Guayaquil; Guayaquil, Ecuador.

\begin{abstract}
Resumen
Objetivo: establecer la eficacia del uso del Plasma Rico en Factores de Crecimiento para ayudar a la consolidación ósea en pacientes que presenten patología tumoral. Los defectos o pérdidas de substancia ósea en el área bucal y maxilofacial son bastante comunes, se deben a diferentes factores como: traumatismos, patología quística o tumoral, atrofias alveolares por pérdidas dentales, periodontopatías, osteorradionecrosis, etc. Para su tratamiento hoy en día, disponemos de varias alternativas como son la distracción ósea, transporte óseo, colgajos osteo-microvascularizados y el plasma enriquecido en factores de crecimiento. En el presente artículo se describen las características histológicas y principios biológicos del plasma rico en factores de crecimiento y su aplicación en 3 casos de cirugía oral y maxilofacial; específicamente patología tumoral. las pérdidas de hueso en el área bucal y maxilofacial representan un verdadero reto de reconstrucción y se debe básicamente a dos situaciones claramente identificadas: 1) la gran cantidad de microorganismos presentes en la cavidad bucal, mismos que en ocasiones contaminan el material injertado con la consecuente pérdida del mismo. 2) las grandes cargas y presiones que deben soportar los injertos durante las fases de masticación. Los estudios realizados en reconstrucción mandibular y maxilofacial por Marx y colaboradores, ' han aportado nuevas pautas en los tratamientos de estos padecimientos, son relevantes sus trabajos con oxígeno hiperbárico, y técnicas quirúrgicas para reconstrucción mandibular con plasma rico en factores de crecimiento.
\end{abstract}

PALABRAS CLAVE: plasma rico en factores de crecimiento, quiste mandibular, reconstrucción mandibular, microorganismos, sistemas de fijación ósea.

Abstract

Objective: establish the efficacy by using Rich Plasma in Growth Factors by helping bone consolidation in patients with tumor pathology. Defects or losses of bone substance in the oral and maxillofacial area are quite common, they are due to different factors such as trauma, cystic or tumor pathology, alveolar atrophies due to dental losses, periodontopathies, osteoradionecrosis, etc. Nowadays treatment has several alternatives such as bone distraction, bone transport, microvascularized bone flaps, and plasma enriched with growth factors. This article describes the histological characteristics and biological principles of rich plasma in growth factors and its application in 3 cases of oral and maxillofacial surgery; specifically, tumor pathology. Bone losses in the oral and maxillofacial area represent a real reconstruction challenge and are due to two identified situations: 1) many microorganisms present in the oral cavity, which sometimes contaminate the grafted material with the consequent loss of it. 2) the high loads and pressures that the grafts must withstand during the chewing phases. Studies carried out on mandibular and maxillofacial reconstruction by Marx et al, 1 have provided new guidelines in the treatment of these conditions, their work with hyperbaric oxygen, and surgical techniques for mandibular reconstruction with plasma rich in growth factors are relevant.

KEYWORDS: plasma-rich growth factors, mandibular cysts, mandibular reconstruction, microorganisms, mouth, rigid bone fixation.

Resumo

Objetivo: estabelecer a eficácia do uso de Plasma Rico em Fatores de Crescimento para ajudar na consolidação óssea em pacientes com patologia tumoral. Defeitos ou perdas de substância óssea na área oral e maxilofacial são bastante comuns, devido a diferentes fatores como: trauma, patologia cística ou tumoral, atrofias alveolares devido a perdas dentárias, periodontopatias, osteorradionecrose, etc. Atualmente, para o seu tratamento, temos várias alternativas, como distração óssea, transporte ósseo, retalhos osteo microvascularizados e plasma enriquecido com fatores de crescimento. Este artigo descreve as características histológicas e os princípios biológicos do plasma rico em fatores de crescimento e sua aplicação em 3 casos de cirurgia oral e maxilofacial; especificamente patologia tumoral. as perdas ósseas na área oral e maxilofacial representam um verdadeiro desafio da reconstrução e se devem basicamente a duas situações claramente identificadas: 1) o grande número de microrganismos presentes na cavidade oral, que às vezes contaminam o material enxertado com a conseqüente perda disso. 2) as altas cargas e pressões que os enxertos devem suportar durante as fases de mastigação. Os estudos realizados na reconstrução mandibular e maxilofacial por Marx et al., 1 forneceram novas diretrizes no tratamento dessas condições, seu trabalho com oxigênio hiperbárico e técnicas cirúrgicas para reconstrução mandibular com plasma rico em fatores de crescimento são relevantes.

PALAVRAS-CHAVE: plasma rico em fatores de crescimento, cistos mandibular, reconstrução mandibular, microrganismos, sistemas de fixação óssea. 
INTRODUCCIÓN

El uso de plasma enriquecido en factores de crecimiento (PRGF), es de las estrategias disponibles el día de hoy en reconstrucciones óseas máxilofaciales, una de las más efectivas; son los responsables de ayudar a modular y aumentar la cicatrización de una herida., ${ }^{1,2,3}$ Estos factores de crecimiento están presentes en las plaquetas, el PRGF debe ser autólogo, no es tóxico y no es inmunorreactivo, acelera las vías de regeneración y cicatrización de una herida, haciendo más resistente el material injertado a infecciones secundarias y al stress masticatorio., ${ }^{4,5,6}$

\section{MATERIAL TEÓRICO}

El PRGF es obtenido de sangre autóloga y mediante la centrifugación se logra la separación celular; la fracción del plasma que conforma aproximadamente $70 \mathrm{ml}$ de volumen, contiene abundante fibrinógeno y factores de coagulación, además de los factores de crecimiento encontrados en las plaquetas. $6,7,8$

Su aplicación clínica para los injertos de hueso requiere la combinación inicial de plasma enriquecido en plaquetas (PEP) con trombina calcificada, lo que va a dar como resultado la formación de un gel; la adición de trombina y calcio al PEP resulta en la activación de la cascada de la coagulación con la conversión de fibrinógeno a fibrina y también la activación y la subsecuente degranulación de las plaquetas. ${ }^{5,15}$ Las plaquetas llegan a ser atrapadas en una red de fibrina, secretando sus contenidos. La matriz de fibrina que resulta del nativo coágulo de fibrina, permite la infiltración celular normal de monocitos, fibroblastos y otras células necesarias para el reestablecimiento de una lesión. ${ }^{8-10} \mathrm{El}$ gel de PEP es añadido al injerto en algunas mezclas. Ésta distribución permite que los factores de crecimiento se distribuyan a través del injerto; a pesar de que la formación de fibrina no es un factor de crecimiento, ésta provee la matriz osteoconductiva necesaria para la regeneración del hueso actuando como un adhesivo que une las células óseas entre sí. ${ }^{11,12}$

Estudios específicos han identificado al menos tres importantes factores de crecimiento en las plaquetas: Factor de crecimiento derivado de plaquetas (PDCF), factor transformador de crecimiento B1 (TGF-B1) y factor transformador de crecimiento B2 (TGF-B2). PDGF parece tener numerosos efectos positivos en el restablecimiento de la lesión, incluyendo mitogénesis, angiogé- nesis y regulación de otras células dando como resultado la activación de funciones osteoblásticas y fibroblásticas; la diferenciación celular y la aceleración de los efectos de los factores de crecimiento en otras células, como los macrófagos. ${ }^{11,12}$

Sus efectos se producen, cuando las moléculas de PDCF se unen a los receptores de membrana celular; esta unión activa una proteína citoplasmática, que activa la expresión genética para las actividades específicas de mitosis, angiogénesis y activación de macrófagos, teniendo como resultado el desbridamiento del sitio de la herida y la promoción de la segunda fase como fuente de factores de crecimiento. 12,13,14

Los PDGF, TGF-B1 y TGFB2 son sintetizados y encontrados en las plaquetas y macrófagos, así como en otros tipos de células ${ }^{7-8}$. Las funciones más importantes de TGF- B1 y TGF-B2 parece ser la quimiotaxis y mitogénesis de los precursores de osteoblastos y la habilidad para estimular su deposición de matriz de colágeno para el tejido conectivo, cicatrización y formación de hueso; además TCF-B inhibe la formación osteoclástica y la reabsorción de hueso. ${ }^{4,18,19}$ ICF-I e IGF-II son factores de crecimiento secretados por los osteoblastos durante la formación de hueso para incrementar el número de osteoblastos y así acelerar la formación de hueso. ${ }^{13,14}$

\section{¿CÓMO SE PRODUCE LA REGENERACIÓN OSEA? \\ El injerto de hueso para reconstruir un defecto de continuidad mandibular, es colocado en un espacio y cubierto por un coágulo de sangre. ${ }^{14}$, 16. Este espacio es hipóxico PO2 (5-10 $\mathrm{mmHg}$ ) y acidótico PH (4-6); contiene plaquetas, leucocitos, glóbulos rojos, fibrina, osteocitos transferidos, osteoblastos endostiales y células progenitoras del hueso esponjoso. ${ }^{13,14}$}

Las células progenitoras de la médula son las primeras células regenerativas del hueso. ${ }^{18}$ Fuera del cierre de la herida quirúrgica, el tejido es fisiológicamente normal PO2 45 - $55 \mathrm{mmHg}$, Ph 7.42 y contiene células mesenquimáticas con capacidad de transformación a otra estirpe de celulas. ${ }^{14,15}$

La iniciación de la regeneración del hueso comienza con la liberación de PDGF y TGF-b de la degranulación plaquetaria en el injerto; PDCF estimula la mitogénesis de las células medulares progenitoras y los osteoblastos endostiales del injerto. ${ }^{4,12}$ Comienza la angiogénesis de muñones 
de capilares inducido por la mitosis celular endotelial. TGF-b inicialmente activa fibroblastos y preosteoblastos aumentando su número y diferenciación hacia osteoblastos funcionantes. ${ }^{15,16}$

La secreción continua de TGF-b influencia a los osteoblastos, para secretar matriz ósea; y a los fibroblastos, para depositar matriz de colágeno, para que sirva de soporte al crecimiento capilar. Todas estas actividades comienzan inmediatamente al cierre de la herida y por el tercer día, los capilares pueden ser vistos que penetran al injerto. ${ }^{14,}{ }^{16}$ La permeabilidad capilar completa del tejido es visto por el día 14 al 17.15,16

El tiempo de vida de una plaqueta en una herida y el período en que sus factores de crecimiento ejercen influencia, es menos de 5 días. ${ }^{14}$ La pro- $^{-}$ longación de la curación y la regeneración ósea está dada por dos mecanismos: ${ }^{15,16}$

1. Aumento y activación de las células progenitoras medulares a osteoblastos, los cuales secretan TGF-b;

2. Quimiotaxis y activación de macrófagos que reemplazan a las plaquetas como fuente primaria de factores de crecimiento después del tercer día.

Los macrófagos son atraídos por acción de PDGFy por el gradiente de oxígeno entre el espacio muerto del injerto y el tejido normal adyacente. ${ }^{15,16}$

MDGF (Factor de crecimiento derivado de macrófagos) y factor angiogénico pueden ser idénticos a PDGF sintetizados por macrófagos en vez de plaquetas; las células progenitoras secretan TGFb para continuar la estimulación propia de formación de hueso como respuesta autócrina..$^{15,16}$

El hueso inicial es desorganizado sin sistemas haversianos e integridad estructural reducida. ${ }^{18}$ Esto es llamado fase I y se desarrolla en las primeras 4 semanas del injerto, luego de ésta, comienza una secuencia de reemplazo, de reabsorción obligatoria lo cual lleva eventualmente a la madurez ósea con arquitectura lamellar y sistemas haversianos, esto es llamado fase II ; en este proceso, un tercer grupo de factores de crecimiento, que no forma parte del PEP, está involucrado, y se denomina proteína morfogenética del hueso (BMP). ${ }^{17,18}$

La liberación de BMP ocasiona nueva formación ósea por las células progenitoras adyacentes, incrementando su número y diferenciación en osteoblastos funcionantes que secretan matriz ósea activamente. ${ }^{14}$ De esta manera el injerto progresa a un hueso funcional maduro el cual se mantiene a través de ciclos de remodelamiento y de reabsorción normal.18,19

\section{REPORTE DE CASOS}

Se realizó colocación de plasma enriquecido en factores de crecimiento en 3 pacientes con diferentes patologías.

\section{CASO 1}

Femenina de 25 años de edad, el dia martes 23 de febrero de 2014, en el Instituto de Cirugía Oral y Maxilofacial, acude a ortodoncista por presentar problemas con su oclusión. En el examen clínico se confirma mal oclusión y se indica estudio radiográfico de rutina. En Rx panorámica se aprecia a nivel de raíces de molares y premolares inferiores izquierdos, mancha radiolúcida de bordes no muy definidos.

Se comprueba dicha patología con tomografía computarizada. Mediante biopsia incisional el estudio histopatológico, reporta el siguiente resultado: mixoma odontogénico. Se programa para resección en bloque de mixoma y reconstrucción inmediata con placa de reconstrucción mandibular, más injerto de cresta iliaca posterior con plasma enriquecido en factores de crecimiento. Usando los criterios de abordaje y protocolo para extirpaciones tumorales mandibulares.

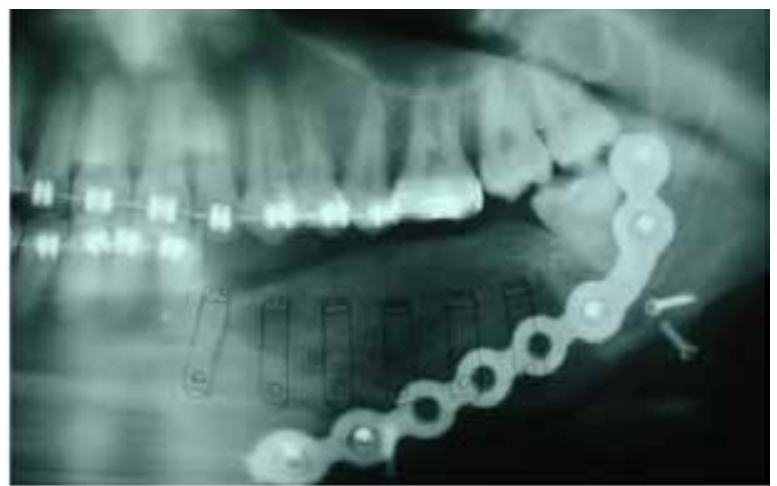

Figura 1. estudio radiográfico para evaluar fijación ósea.

Fuente: Instituto de Cirugía Oral y Maxilofacial, Guayaquil.

Después de 15 días se elimina fijación interdentomaxilar y se realiza seguimiento mensual durante 6 meses, tiempo en el cual se aprecia consolidación adecuada del injerto. Figura 1. Al séptimo mes de resección se realiza segunda intervención quirúrgica; se procede a realizar el retiro de placa de reconstrucción mandibular 
y la colocación de 5 implantes óseo-integrados marca RESTORE HA de $4 \mathrm{~mm}$ de diámetro por $15 \mathrm{~mm}$ de longitud. Después de 6 meses de la colocación de los implantes se remite a rehabilitación oral para confección de prótesis dental. Se realizará controles a los 15 años.

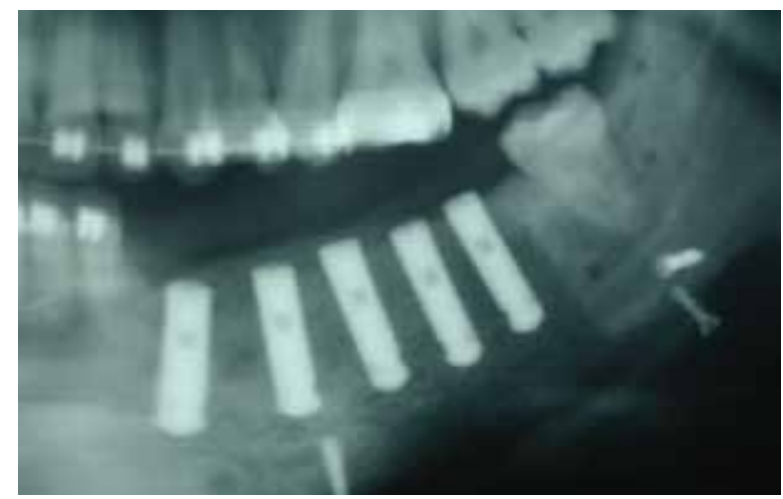

Figura 2. Radiografía con zonas radiopacas compatibles con implantes dentales. Fuente: Instituto de Cirugía Oral y Maxilofacial, Guayaquil.

CASO 2

El día 21 de diciembre de 2014 acude al Instituto de Cirugía Oral y Maxilofacial un paciente masculino de 23 años de edad con tercer molar inferior derecho retenido y radiográficamente presenta imagen radiolúcida de grandes proporciones.

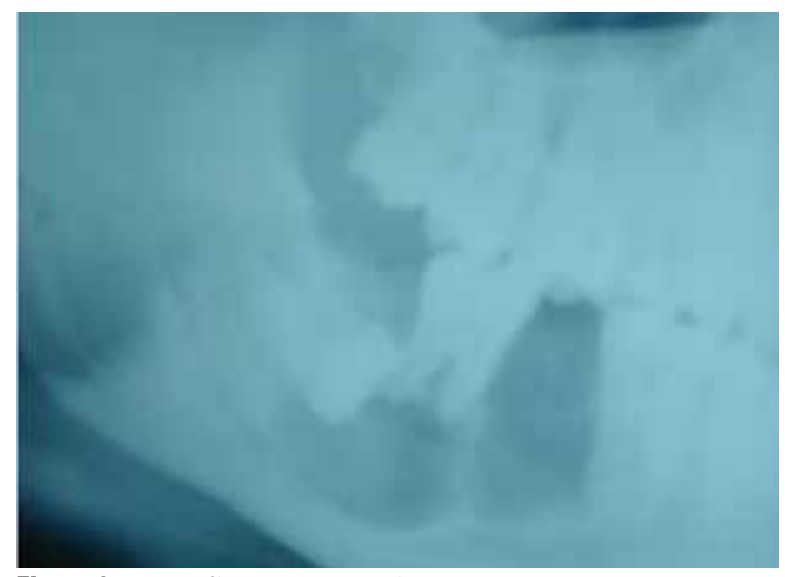

Figura 3. Radiografía con zonas radiolúcidas en zona posterior. Fuente: Instituto de Cirugía Oral y Maxilofacial, Guayaquil.

Clínicamente el segundo molar presenta movilidad. Se realiza biopsia incisional. El examen histológico da como resultado el diagnóstico de ameloblastoma plexiforme. Se realiza la exodoncia de dientes comprometidos y la resección en bloque con márgenes de seguridad así como también la reconstrucción inmediata del defecto con injerto de hueso iliaco posterior mezclado con plasma enriquecido en factores de crecimiento. Al mes de la cirugía se aprecia claramente la fase II de cicatrización ósea en la cual se aprecia zona de regeneración.
Dos meses después es visible la proliferación ósea a expensas del tejido transplantado; es visible también imagen radiolúcida a nivel de premolar No. 45.

El paciente presenta necrosis pulpar de premolar y se remite a odontóloga que no realiza tratamiento de endodoncia, dando como resultado un absceso apical y por consiguiente propagación del cuadro infeccioso a través del hueso neoformado. Se realiza exodoncia de premolares, secuestrectomía, curetaje y se decide colocar una placa de reconstrucción más superior para afianzar el tejido neoformado. 3 meses después se toma rx de control y se aprecia que todavía no se tiene una osificación completa.

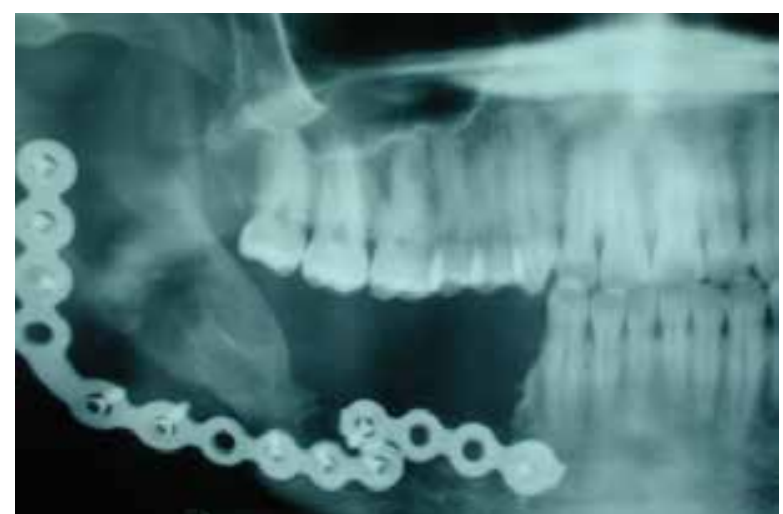

Figura 4. radiografía de control con radiopacidades compatibles con placas de fijación ósea.

Fuente: Instituto de Cirugía Oral y Maxilofacial, Guayaquil.

CASO 3

El 23 de mayo de 2014, acude al Instituto de Cirugía Oral y Maxilofacial un paciente de 60 años que presenta imagen radiolúcida a nivel de tercer molar inferior izquierdo. El resultado histopatológico demuestra que se trata de quiste dentígero. Se programa quistectomía más relleno del defecto con injerto de hueso de banco mezclado con plasma enriquecido en plaquetas. Se fijará nuevamente la cortical con miniplaca y tornillos de titanio. Figura 5

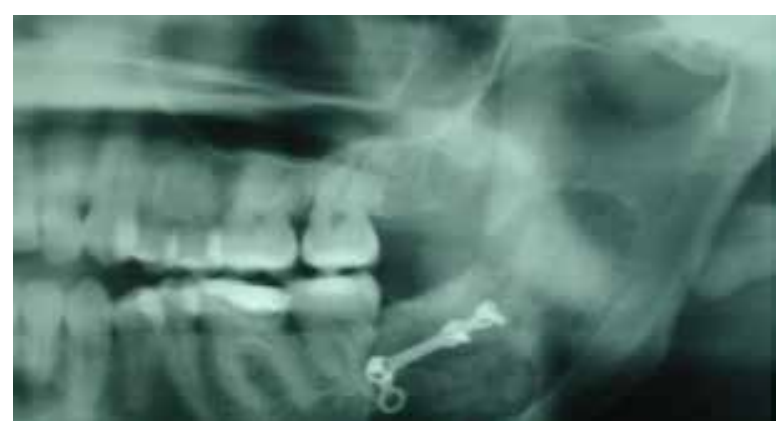

Figura 5. Radiografía con presencia de radiolucides con bordes definidos compatible con quiste mandibular. Fuente: Instituto de Cirugía Oral y Maxilofacial, Guayaquil 
La imagen corresponde a una semana posterior a cirugía. A los 30 días se realiza Rx. de control donde es claramente visible la consolidación total del injerto. A los 6 meses de cirugía no hay evidencia de recidiva quística y cicatrización total del injerto, figura 6 .

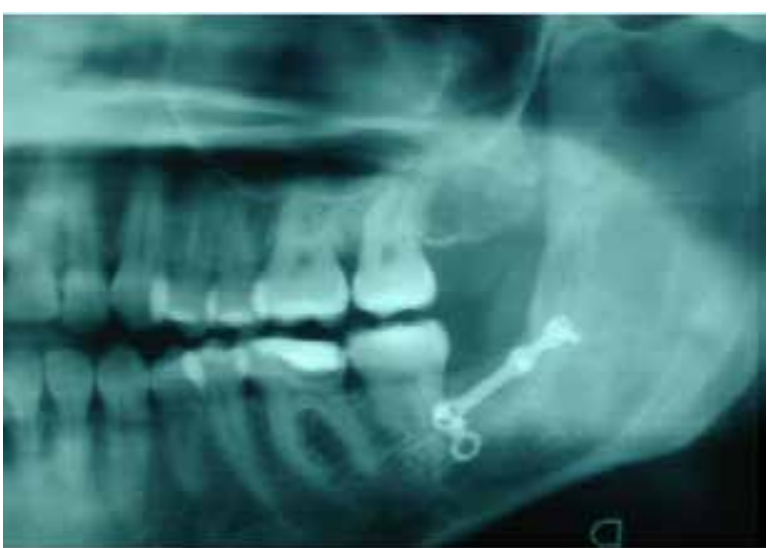

Figura 6. radiografía donde se aprecia osificación ósea y radiopacidad compatible con mini placa de osificación mandibular.

Fuente: Instituto de Cirugía Oral y Maxilofacial, Guayaquil.

\section{CONCLUSIONES}

Las cirugías de reconstrucción en los maxilares presentan algunos retos para el cirujano, ambiente bacteriológico amplio, grandes cargas mecánicas y alta demanda estética.

Los problemas inherentes a reemplazar, radican en que tienen que soportar aparatos protésicos dentales y verse como piezas dentales naturales.

Los problemas de acoplamiento de los injertos están reportados en la literatura, como por ejemplo el aumento vertical de rebordes, que por la fuerza gravitacional, los resultados eran muy pobres.

El PRGF ha permitido a manera de un gel cohesivo, mantener la verticalidad de los injertos colocados, permitiendo superar la disgregación del injerto y por ende ausencia de reconstrucción vertical.

El costo económico es importante en América Latina, se promueve el uso de PRGF mucho más económico, para obtener cicatrización más rápida y con menos exposición del injerto, como sucede con mallas de titanio y membranas reforzadas de colágeno al ambiente bucal en la fase de integración.

Otra herramienta en el arsenal terapéutico para promover una cicatrización más acorde con las necesidades de los clínicos.
En los casos presentados se logró una rápida consolidación de los injertos colocados; hay que hacer énfasis que el PRGF es y debe ser exclusivamente autólogo. La obtención del mismo no requiere equipos altamente sofisticados, debido a que su preparación radica en la centrifugación y separación de los elementos sanguíneos y la colocación de substancias que promuevan la formación de gel.

Se recomienda establecer protocolos para el uso del PRGF para las diferentes áreas médicas y odontológicas que pueden necesitar de una terapia biológica en el cierre y cicatrización de heridas.

\section{REFERENCIAS BIBLIOGRÁFICAS}

1. Marx RE, Carlson ER, Eichstaedt RM, et al. Plateletrich plasma. Growth factor enhancement for bone grafs. Oral Surgery Oral Medicine Oral Patology Oral radiology 1998; 85: 638-646

2. Marx RE, Ehler Wj, Peleg M. Mandibular and facial reconstruction: rehabilitation of the head and neck cancer patient. Bone 1996; 19 Suppl 1: 595- 625

3. Anitua E. The use of plasma-rich growth factors (PRGF) in oral surgery. Pract Proced Aesthet Dent. 2001; 13: 487-93.

4. Gonshor A. Técnicas para producir plasma rico en plaquetas y concentrado plaquetario: Antecedentes y proceso. Revista Internacional de Odontología Restauradora y Conservadora. 2002; 6: 583-93.

5. Anitua E. Un nuevo enfoque en la regeneración ósea. Plasma rico en factores de crecimiento (P.R.G.F). Editorial: Puesta al día publicaciones, S.L. 2000 Vitoria-España.

6. Romo-Simón L. y cols. Plasma rico en factores de crecimiento óseo en quistes maxilares y sobre implantes. Resultados preliminares. RCOE. 2001; 6: 48.

7. Dugrillon A y cols. Autologous concentrated plateletrich plasma (CPRP) for local application in bone regeneration. Int. J. Oral Maxillofac.Surg. 2002; 31: 615-9.

8. Marx RE. Platelet-Rich Plasma: A source of multiple autologous growth factors for bone grafts. Tissue Engeneering: Aplicationns in Maxillofacial Surgery and Periodontics. Editorial: Quintessense Books.1999, Illinois -Estados Unidos.

9. Mancuso, Bennion, Hull. Platelet rich plasma: A preliminary report in routine impacted third molar surgery and the prevention of alveolar osteitis. J Oral Maxillofac Surg. 2003; 61: 36.

10. Okuda K, Kawase T, Momose M, Murata M, Saito Y, Suzuki H, Wollf LF. Platelet rich plasma contains high levels of platelet derived growth factors and 
transforming growth factor beta and modulates the proliferation of periodontal related cells in vitro. J Periodontal. 2003; 74: 849-57.

11. Camargo PM, Lekovic V, Weinlander M, Vasilic N, Madzarevic M, Kenney EB. Platelet rich plasma and bovine porous bone mineral combined with guided tissue regeneration in the treatment of bony defects in humans. J Periodontol Res. 2002; 37: 300-6.

12. Fuerst G. y cols. Enhanced Bone-to- Implant Contact by Plateled-released Growth Factors in Mandibular Cortical Bone: A histomorphometric Study in minipigs. Int J Oral Maxillofac Implants. 2003; 18: 685-90.

13. Wiltfang J, Schlegel KA, Schultze-Mosgau S, Nkenke E, Zimmerman R, Kessler P. Sinus floor augmentation with beta-tricalciumphosphate: Does platelet rich plasma promote its osseous integration and degradation? Clin Oral Implants Res. 2003; 14: 213-8.

14. B-H Choi, S-J Zhu, B-Y Kim, J-Y Huh, S-H Lee, J-H Jung. Effect of platelet-rich plasma (PRP) concentration on the viability and proliferation of alveolar bone cells: An in vitro study. Int J Oral Maxillofac. Surg 2005; 34: 420-4.
15. Marx RE. Platelet rich plasma (PRP): What is PRP and what is not PRP? Implant Dent. 2001; 10: 225-8.

16. Cornelini R. et al. Vascular Endothelial Growth Factor and Microvessel Density around healthy and failing implants.Int J Oral Maxillofac Implants, 2001; 16: 389-93.

17. Kim S. et al. A comparative study of osseointegration of Avana Implants in a Desmineralized FreezeDried Bone alone or with Platelet-Rich Plasma. J Oral Maxillofac Surg, 2002; 60: 1018-25.

18. Anitua E. Expansión de cresta con osteotomos: Estado actual. Utilización de plasma rico en factores de creci-miento (P.R.G.F). Rev Esp Cirug Oral y Maxilofacial, 2001; 23: 158-62.

19. Landesberg R. et al. Quantification of Growth factor levels using a simplified method of Platelet-Rich Plasma Gel Preparation. J Oral Maxillofac Surg, 2000; 58: 297-300. 